\title{
Strukturwandel und Zukunft der Interessenvertretung im Handwerk
}

\author{
Das Handwerk ist mit 4,9 Mio. Beschäftigten und ca. 580.000 Unternehmen einer der \\ bedeutendsten Wirtschaftsbereiche in Deutschland. Derzeit durchläuft es einen struktu- \\ rellen Wandel, der sich in Konzentrations- und Polarisierungstendenzen und einem Ver- \\ schwimmen der Grenzen zwischen den einzelnen Gewerken des Handwerks und zwi- \\ schen Handwerk und Industrie niederschlägt. Gleichzeitig haben sich das tarifpolitische \\ Umfeld im Handwerk dramatisch verändert, und damit auch die Rahmenbedingungen \\ für die Interessenvertretung in diesem mitbestimmungspolitisch nach wie vor wenig er- \\ schlossenen Bereich. Der Beitrag beschreibt die damit verbundenen neuen Aufgaben \\ und Probleme für Betriebsräte und Gewerkschaft. ${ }^{1}$
}

STEFANIE WEIMER

\section{Einleitung}

Der Beitrag beschreibt den Strukturwandel im Handwerk: die Polarisierung der Betriebsstrukturen, deutliche Konzentrationsprozesse und gleichzeitig eine Dezentralisierung der tarifpolitischen Rahmenbedingungen. Vor diesem Hintergrund stellt er die Frage, wie sich diese veränderten strukturellen Rahmenbedingungen auf der Ebene der betrieblichen und überbetrieblichen Interessenvertretung im Handwerk niederschlagen. Er kommt zu dem Schluss, dass die Anforderungen an die Betriebsräte im Handwerk in vielerlei Hinsicht komplexer werden und der Aufbau funktionierender Mitbestimmungsstrukturen in diesem nach wie vor mitbestimmungspolitisch unzureichend erschlossenen Bereich ohne massive Unterstützung durch die Gewerkschaft kaum möglich sein wird. Dies wirft nicht zuletzt die Frage nach einer Neuverteilung der gewerkschaftlichen Betreuungsressourcen zwischen Handwerk und Industrie auf.

\section{Der Strukturwandel im Handwerk...}

Betrachtet man die Entwicklung des Handwerks in den letzten 15 Jahren im Spiegel der letzten beiden Handwerkszählungen von 1995 und 2008, ${ }^{2}$ so zeigt sich als Grundtendenz (vgl. Tabelle 1) ein deutlicher Zuwachs an Unternehmen (um gut $20 \%$ ) und eine Zunahme des Handwerksumsatzes (um ca. $15 \%$ ) bei gleichzeitigen massiven Verlusten an Beschäftigten. Das Handwerk hat in den letzten Jahren über eine Million Arbeitsplätze verloren.

Dieser Trend ist in den einzelnen Betriebsgrößenklassen keinesfalls einheitlich verlaufen: So geht der Unternehmenszuwachs ausschließlich auf das Konto der Kleinstbetriebe Auswirkungen der Reform der Handwerksordnung 2004 mit

1 Dieser Beitrag fußt auf Ergebnissen eines von der HansBöckler-Stiftung geförderten, von 2010 bis 2012 durchgeführten Forschungsprojekts (Projektnr. 2009-315-2). Die Ergebnisse wurden in klassischen Branchen des Metallhandwerks - Kfz-Gewerbe, Sanitär-, Heizungs- und Klimahandwerk (SHK) sowie Elektrohandwerk - gewonnen, ähnliche strukturelleTendenzen finden sich aber auch in anderen Handwerksbranchen.

2 Da zwischen den beiden Handwerkszählungen eine grundlegende Umstellung der Erhebungsmethode - von einer Unternehmensbefragung auf die Sekundärauswertung des Unternehmensregisters - sowie eine weitreichende Reform der Handwerksordnung stattgefunden hat, sind die Daten aus beiden Zählungen nur eingeschränkt vergleichbar. Um dennoch einen Vergleich zu ermöglichen und somit Strukturentwicklungen nachvollziehen zu können, wird im Folgenden auf korrigierte Daten der Handwerkszählung 2008 zurückgegriffen. Sie basieren auf Berechnungen des Volkswirtschaftlichen Instituts für Mittelstand und Handwerk der Universität Göttingen (Müller 2012). 
ihrer Ausweitung der zulassungsfreien Handwerke -, während alle anderen Betriebsgrößenklassen Rückgänge aufweisen. Am stärksten ist der Rückgang bei den mittleren Betriebsgrößenklassen mit zehn bis 19 Beschäftigten ausgeprägt, am geringsten bei den Großbetrieben mit über 50. Ein ähnliches Bild zeigt sich bei der größenspezifischen Verteilung der Beschäftigtenentwicklung: Außer den Kleinstbetrieben haben alle Größenklassen Beschäftigtenverluste hinnehmen müssen, besonders ausgeprägt in den mittleren Betriebsgrößenklassen. Umsatzzuwächse verzeichnen nur die Kleinstbetriebe - Effekt ihrer starken Zunahme - und die „Großen“ mit über 50 Beschäftigten.

Tabelle 2 zeigt, wie sich im Zeitablauf die Verteilung von Beschäftigten, Umsatz und Unternehmen auf die Größenklassen verändert hat. Der Anteil der Kleinstbetriebe an den Unternehmen ist in den letzten 15 Jahren von rund 47 auf $67 \%$ gestiegen. Entsprechend konnten sie ihren Anteil an Beschäftigten ausweiten, während die mittleren Größenklassen Beschäftigte verloren. Etwa ein Drittel der Beschäftigten arbeitete 2008 in Großbetrieben, hier ergaben sich keine Veränderungen. Beim Umsatz haben die Großen gewonnen: Sie erwirtschafteten 2008 fast $40 \%$ des Handwerksumsatzes.

Zugespitzt lässt sich aus den statistischen Daten ableiten, dass sich im Handwerk eine Tendenz zur Polarisierung vollzieht, durch die sich drei Segmente herausbilden:

(1) Auf der einen Seite findet man viele Klein- und Kleinstbetriebe mit oft prekären Beschäftigungsbedingungen, ein großer Teil von ihnen als Ein-Personen-Unternehmen, darunter viele Scheinselbstständige. Dieser Bereich dürfte für die Gewerkschaft kaum zu organisieren sein, ist aber dennoch relevant, weil von ihm ein erheblicher Preis- und Lohndruck auf die anderen Bereiche des Handwerks ausgeht.

(2) Auf der anderen Seite steht die Gruppe der besonders beim Umsatz prosperierenden Großunternehmen. Eine Untersuchung der Zeitschrift handwerk-magazin (2011) identifizierte 2011 1.000 Unternehmen dieses sogenannten „modernen Großhandwerks“ als ,innovativen Bereich der Handwerksbranche" mit einem Gesamtumsatz von 35,7 Mrd. $€$, verteilt auf ein Spektrum, das von Unternehmen mit 13 Mio. $€$ Umsatz bis zu solchen mit 1,1 Mrd. € Umsatz reicht. Solche Unternehmen finden sich insbesondere im Baugewerbe, im Nahrungsmittelhandwerk und im Gebäudereinigerhandwerk, aber auch in klassischen Branchen aus dem Organisationsbereich der IG Metall, so dem Kfz-Gewerbe, dem SHK-Handwerk und dem Elektrohandwerk.

(3) Dazwischen liegt die tendenziell sich ausdünnende breite Mitte, der klassische Kernbereich der mittelgroßen Betriebe des Handwerks, der zwar immer noch einen großen Teil des Handwerks ausmacht, aber zunehmend unter Druck gerät: durch die Unterbietungskonkurrenz der Kleinstbetriebe, aber auch durch die Konkurrenz der Großen, die in seine traditionellen lokalen Geschäftsfelder eindringen.
TABELLE 1

\section{Grunddaten im Handwerk nach Beschäftigtengrößenklassen 1994/95 und 2008}

Angaben in absoluten Zahlen und in Prozent

\begin{tabular}{|c|c|c|c|}
\hline & 1995 & 2008 (ergänzt) & $\begin{array}{c}\text { Veränderung } \\
1994 / 95 \text { zu } 2008\end{array}$ \\
\hline \multicolumn{4}{|l|}{ Unternehmen } \\
\hline unter 5 & 265.118 & 454.704 & $71,5 \%$ \\
\hline $5-9$ & 155.020 & 121.075 & $-21,9 \%$ \\
\hline $10-19$ & 89.893 & 63.682 & $-29,2 \%$ \\
\hline $20-49$ & 42.159 & 30.689 & $-27,2 \%$ \\
\hline 50 und mehr & 14.717 & 12.029 & $-18,3 \%$ \\
\hline Handwerk gesamt & 566.907 & 682.179 & $20,3 \%$ \\
\hline \multicolumn{4}{|l|}{ Tätige Personen } \\
\hline unter 5 & 623.277 & 807.338 & $29,5 \%$ \\
\hline $5-9$ & 1.023 .455 & 791.067 & $-22,7 \%$ \\
\hline $10-19$ & 1.193 .100 & 843.703 & $-29,3 \%$ \\
\hline $20-49$ & 1.221 .761 & 896.090 & $-26,7 \%$ \\
\hline 50 und mehr & 2.016 .979 & 1.673 .724 & $-17,0 \%$ \\
\hline Handwerk gesamt & 6.104 .932 & 5.021 .182 & $-17,8 \%$ \\
\hline \multicolumn{4}{|l|}{ Umsatz } \\
\hline unter 5 & 29.216 .023 & 50.179 .530 & $71,8 \%$ \\
\hline $5-9$ & 60.449 .335 & 57.462 .265 & $-4,9 \%$ \\
\hline $10-19$ & 83.432 .126 & 75.365 .990 & $-9,7 \%$ \\
\hline $20-49$ & 99.765 .420 & 97.458 .832 & $-2,3 \%$ \\
\hline 50 und mehr & 135.643 .527 & 183.857 .256 & $3,5 \%$ \\
\hline Handwerk gesamt & 410.717 .239 & 471.302 .093 & $14,8 \%$ \\
\hline
\end{tabular}

Anmerkung: Summe der Einzelwerte bei tätigen Personen und Umsatz entspricht nicht der Gesamtsumme wegen Geheimhaltung.

Quelle: Müller (2012, S. 136).

Dieser Prozess der Polarisierung und Konzentration ist in den Branchen des Handwerks unterschiedlich ausgeprägt. Tabelle 3 zeigt, dass es zum einen Branchen mit reinen Dekonzentrationsprozessen gibt, d.h. einer Zunahme der Kleinstbetriebe bei gleichzeitiger Abnahme von Großbetrieben. Dies ist im Bau- und Ausbaugewerbe der Fall. Besonders ausgeprägte Konzentrationsprozesse zeigen sich zum anderen im Lebensmittelgewerbe, im Kfz-Gewerbe und im Gesundheitsgewerbe. Insbesondere die letzteren beiden weisen auch eine sehr ausgeprägte brancheninterne Polarisierungstendenz auf: Es wächst sowohl die Zahl der Kleinstbetriebe als auch die der Großen, während die mittelgroßen Betriebe ausdünnen.

Die amtliche Handwerkszählung bildet allerdings den Konzentrationsprozess im Handwerk nur unzureichend ab. Das hat verschiedene Gründe. So differenziert die amtliche Handwerksstatistik oberhalb 50 Beschäftigten und 5 Mio. Umsatz nicht mehr weiter in Größenklassen. Die Erfassungseinheit ist das Unternehmen als rechtliche Einheit, in vielen Unternehmensgruppen haben die einzelnen Betriebe aber die Rechtsform selbstständiger GmbHs, die unter 
TABELLE 2

Zusammensetzung des Handwerks nach Beschäftigtengrößenklassen 1994/95 und 2008

Angaben in Prozent

\begin{tabular}{|c|c|c|c|c|c|c|}
\hline \multirow[b]{2}{*}{ Personen } & \multicolumn{2}{|c|}{ Unternehmen } & \multicolumn{2}{|c|}{ Tätige Personen } & \multicolumn{2}{|c|}{ Umsatz } \\
\hline & 1995 & 2008 (ergänzt) & 1994 & 2008 (ergänzt) & 1994 & 2008 (ergänzt) \\
\hline unter 5 & 46,8 & 66,7 & 10,3 & 16,1 & 7,2 & 10,8 \\
\hline $5-9$ & 27,3 & 17,7 & 16,8 & 15,8 & 14,8 & 12,4 \\
\hline $10-19$ & 15,9 & 9,3 & 19,6 & 16,8 & 20,4 & 16,2 \\
\hline $20-49$ & 7,4 & 4,5 & 20,1 & 17,9 & 24,4 & 21,0 \\
\hline 50 und mehr & 2,6 & 1,8 & 33,2 & 33,4 & 33,2 & 39,6 \\
\hline Handwerk gesamt & 100,0 & 100,0 & 100,0 & 100,0 & 100,0 & 100,0 \\
\hline
\end{tabular}

Quelle: Müller (2012, S. 137).

TABELLE 3

Veränderung der Zahl der Handwerksunternehmen in den einzelnen Beschäftigtengrößenklassen zwischen 1995 und 2008 nach Gewerbegruppen

Angaben in Prozent

Veränderungen 1995-2008

\begin{tabular}{|c|c|c|c|c|c|c|}
\hline Gewerbegruppe & unter 5 Personen & 5-9 Personen & 10-19 Personen & 20-49 Personen & 50 und mehr Pers. & Gesamt \\
\hline Bauhauptgewerbe & 180,6 & $-1,4$ & $-41,6$ & $-58,7$ & $-63,3$ & 22,9 \\
\hline Ausbaugewerbe & 103,1 & $-27,0$ & $-36,5$ & $-37,0$ & $-33,4$ & 31,5 \\
\hline Handwerke für den gewerblichen Bedarf & 51,0 & $-18,9$ & $-5,6$ & 17,1 & 7,7 & 21,1 \\
\hline Kraftfahrzeuggewerbe & 54,0 & $-11,0$ & $-14,9$ & $-11,1$ & 24,0 & 18,0 \\
\hline Lebensmittelgewerbe & 27,2 & $-48,6$ & $-33,2$ & $-5,5$ & 34,2 & $-32,9$ \\
\hline Gesundheitsgewerbe & 69,5 & 3,5 & $-12,5$ & $-9,9$ & 31,4 & 24,3 \\
\hline Handwerke für den privaten Bedarf & 38,5 & $-19,3$ & $-16,1$ & $-9,4$ & $-9,0$ & 22,5 \\
\hline Handwerk gesamt & 71,5 & 21,9 & $-29,2$ & $-27,2$ & $-18,3$ & 20,3 \\
\hline
\end{tabular}

Quelle: Müller (2012, S. 146).

dem Dach einer Holding zu einer Unternehmensgruppe zusammengefasst sind. Auch die Praxis, Umsätze sogenannter steuerrechtlicher Organschaften (gemeinsam besteuerte Tochterunternehmen eines Konzerns, dahinter verbergen sich in der Regel Unternehmensgruppen) künstlich auf die Einzelbetriebe herunterzurechnen, trägt dazu bei, dass die Zahl von Handwerksunternehmen mit sehr hohen Umsätzen systematisch unterschätzt wird. Im Jahr 2008 waren immerhin $4 \%$ der steuerpflichtigen Unternehmen des Handwerks Teil einer steuerrechtlichen Organschaft, also eines Konzerns. Die Frage stellt sich also auf qualitativer Ebene: Was sind die Antriebskräfte des Konzentrationsprozesses im Handwerk? Welche Geschäftsmodelle und welche Unternehmen entstehen hier?

\section{3. ... und seine Antriebskräfte}

Ein typischer Prozess ist das Breitenwachstum durch Ausweitung der Geschäftsfelder auf zusätzliche handwerkliche und nicht-handwerkliche Tätigkeiten. Dieses Wachstum kann marktgetrieben sein; es kann gefördert werden durch die Kundennachfrage nach „Angeboten aus einer Hand“, aber auch durch technologische Entwicklungen, z. B. den Technologieschub in der Gebäudetechnik, wo verschiedene Technikfelder wie Elektronik, Heizungs- und Klimatechnik, IT und damit auch verschiedene Branchen und Berufsfelder zusammenwachsen. Ein solches Breitenwachstum, das verschiedene klassische handwerkliche Tätigkeitsfelder betrifft, findet sich z. B. im Zuge der Ausbildung der Branche Facility Management (FM). So weiten etwa Unternehmen des Gebäudereinigerhandwerks ihr Tätigkeitsspektrum vom 
infrastrukturellen FM (Reinigung, Bewachung, Catering) auf hochwertige Dienstleistungen wie die technische Gebäudeausstattung und -instandhaltung aus.

Motive für diese Ausweitung sind die höhere Wertschöpfungsintensität dieser Dienstleistungen sowie der Druck der Kunden auf Full-Service-Angebote. Das notwendige Knowhow und die erforderliche Präsenz in der Fläche erreichen diese Unternehmen häufig durch den Aufkauf einschlägiger Handwerksunternehmen und durch den Kauf einschlägiger Dienstleistungsbereiche von Industrieunternehmen. Das noch relativ junge Geschäftsfeld FM ist ein Flickenteppich unterschiedlicher Branchenherkunft: Neben dem Gebäudereinigerhandwerk rekrutieren sich die großen „Player“ der Branche aus dem Bauhandwerk, z. T. großen Baukonzernen.

Technische Gebäudeausstattung und -instandhaltung erfordern Kundennähe und die Leistungserbringung findet an stark wechselnden Einsatzorten statt. Die Folge ist, dass hier Unternehmensgruppen entstehen, die länderübergreifend, oft bundesweit tätig sind, vor Ort aber durch kleinteilige Standortstrukturen, z. B. in Form regionaler Niederlassungen, gekennzeichnet sind und eine mobile Arbeitsorganisation haben.

Ein weiterer Entstehungshintergrund von großen Unternehmensgruppen im Handwerk sind Dezentralisierungsprozesse in großen Fertigungsunternehmen durch die Auslagerung von Dienstleistungen oder Fertigungsschritten. Dies spielt im Metallhandwerk vor allem in der sich neu formierenden Branche der industrienahen bzw. produktionsnahen Dienstleistungen eine Rolle. Industrienahe Dienstleistungen umfassen Tätigkeiten wie Montage und Demontage, Reinigung, Reparatur und Instandhaltung von Produktionsanlagen und Infrastruktur (z. B. überregionale Leitungsnetze, gebäudetechnische Anlagen) bis hin zur Übernahme von einzelnen Fertigungsschritten wie Montageaufgaben. Die hier angesprochenen Tätigkeitsfelder werden von Unternehmen des Elektrohandwerks, des Metallbauhandwerks und der heizungs- und klimatechnischen Handwerke abgedeckt. Auslagernde Industrien sind vor allem die Automobilindustrie, die Eisen- und Stahlindustrie, der Maschinen- und Anlagenbau sowie Prozessindustrien wie die Chemische Industrie, die Nahrungs- und Genussmittelindustrie oder die Petrochemie.

Ein zentrales Motiv für diese Auslagerung von Industriedienstleistungen ist die Realisierung von Kosten- und Wettbewerbsvorteilen durch Vergabe an preislich günstigere externe Anbieter. Solche Kostenvorteile werden nicht zuletzt dadurch realisiert, dass die Anbieter solcher Dienstleistungen häufig dem Handwerk mit seinen niedrigeren Lohnstrukturen zuzurechnen sind. So werden ehemals konzerneigene Dienstleistungs- und Instandhaltungsabteilungen nach der Auslagerung aus dem Konzern in das Handwerk und damit in den billigeren Handwerkstarif transferiert. Nicht selten bildet dieser Transfer die Vorstufe zur Weiterveräußerung des Unternehmensteils.

Auch hier führt die Notwendigkeit der Kundennähe und der Flexibilität der Leistungserbringung zu dezentralen, breit gestreuten und klein dimensionierten Standortstrukturen in Form kleinerer Niederlassungen oder Stützpunkte; häufig wird die Leistung direkt im Werk des Kunden erbracht.

Nicht zuletzt sind es Konzentrationsprozesse innerhalb einzelner Handwerksbranchen durch Fusionen, Aufkäufe und Filialisierungsprozesse, die zu Großbetrieben des Handwerks führen, wobei die Antriebsfedern solcher brancheninternen Konzentrationsprozesse branchenspezifisch sehr unterschiedlich sein können. Im Fall des Kfz-Gewerbes, das immer mehr von überregionalen Autohandelsgruppen dominiert wird, wurde ein rasanter Konzentrationsprozess eingeleitet durch das Zusammenwirken der Liberalisierung der wettbewerbspolitischen Rahmenbedingungen der Branche durch die EU, einer Ertragskrise und von Bestrebungen der Hersteller, ihr Vertriebs- und Servicenetz aus Kostengründen zu straffen. Für all diese Prozesse ist kennzeichnend,

- dass die neuen Geschäftsfelder auch vielen mittleren Unternehmen des Handwerks Marktchancen bieten. Es können auch normale Handwerksunternehmen durch internes Wachstum und bundesweiten Aufkauf anderer Handwerksunternehmen zu Unternehmensgruppen heranwachsen. So sind in den letzten Jahren neben den großen Handwerkskonzernen auch eine Reihe quasi mittelständischer Unternehmensgruppen des Handwerks entstanden, mit 500 bis 1.500 Beschäftigten und oft mit regionalen Schwerpunkten. - dass zunehmend handwerksexterne Anbieter, z. B. Anlagenbauer, in diese Handwerksbereiche eindringen, denn industrienahe Dienstleistungen und technisches Gebäudemanagement gelten als Wachstumsmarkt und, da es sich um hochwertige Dienstleistungen handelt, als lukratives Geschäftsfeld. Dies lockt auch externe Investorengruppen an. Sie kaufen auf diesen Geschäftsfeldern tätige Handwerksunternehmen, die insolvent werden oder ohne Nachfolge bleiben, auf und fassen sie zu Unternehmensgruppen zusammen. Auch der demografische Wandel ist damit ein nicht zu unterschätzender Treiber dieses Strukturwandels im Handwerk, der in Zukunft verstärkt Investorenkapital ins Handwerk bringen wird.

- dass der Anbietermarkt in diesen neuen Geschäftsfeldern des Handwerks sich durch eine hohe Dynamik von Abspaltungen, Aufkäufen, Zusammenschlüssen und Wiederverkäufen von Unternehmen und Unternehmensteilen auszeichnet.

\section{Veränderung der tarifpolitischen Landschaft}

Nicht nur die Betriebsstrukturen, auch das tarifpolitische Umfeld hat sich im Handwerk in den letzten Jahren gravierend verändert. Der Organisationsgrad der Handwerksunternehmen in den Arbeitgeberverbänden ist stark gesunken. In den alten Ländern sind nach Schätzungen der Arbeitnehmervizepräsidenten der Kammern (im Durchschnitt 
der Kammern) nur noch 40 bis $45 \%$ der Betriebe Mitglied einer Innung, in den neuen Bundesländern nur noch ca. 10 bis $15 \%$. Die Flucht der Unternehmen aus den Arbeitgeberverbänden, verbunden mit der Gründung sogenannter OTVerbände, hat zu einer Erosion des Flächentarifvertrags in weiten Bereichen des Handwerks geführt. In vielen großen Branchen des Metallhandwerks und in manchen Bundesländern existiert kein Flächentarifvertrag mehr. Die Zahl tarifloser Betriebe nimmt zu, insbesondere in den neuen Bundesländern. Dort, wo noch Flächentarifverträge gelten, stehen dahinter oftmals nur noch wenige größere Unternehmen oder kleinere Tarifgemeinschaften. Zudem spielt im Handwerk die Konkurrenz von Niedriglohngewerkschaften, wie z. B. den sogenannten Christlichen Gewerkschaften, eine bedeutende Rolle: Es gibt im Handwerk Branchen, in denen die Landesinnungsverbände, traditioneller Tarifpartner der DGB-Gewerkschaften, nur noch Flächentarifverträge mit den „Christlichen“ abschließen. Im Handwerk haben infolgedessen Haustarifverträge zuungunsten des Flächentarifs stark an Bedeutung gewonnen: Allein im Organisationsbereich der IG Metall betrug die Zahl der im Handwerk abgeschlossenen Haustarifverträge Anfang 2011 schätzungsweise 1.000 (IG Metall Vorstand 2011).

Eine gravierende Folge des Strukturwandels im Handwerk hin zu zunehmend gewerkeübergreifenden Tätigkeitsfeldern und Unternehmensgruppen mit überregionaler Ausbreitung besteht darin, dass in solchen Unternehmensgruppen oft nicht mehr von einer einheitlichen Tarifbindung für das gesamte Unternehmen ausgegangen werden kann. Historisch gewachsene Tarifbindungen nach der Primärindustrie, aus der die Gruppen entstanden sind, oder nach Herkunft der irgendwann zugekauften Betriebe bleiben bestehen. In einer Unternehmensgruppe gelten daher oft nicht nur unterschiedliche Branchentarifverträge des Metallhandwerks in je unterschiedlicher regionaler Ausgestaltung, sondern auch Tarifverträge anderer Gewerkschaften, die einander nicht selten konkurrierend gegenüberstehen. ${ }^{3}$

Um ihre Tarifbedingungen unternehmensweit zu harmonisieren, schließen auch die Unternehmensgruppen vermehrt Haustarifverträge ab. Unterstützt werden sie dabei von Unternehmensverbänden, für die das Angebot, „maßgeschneiderte" Haustarifverträge zu entwickeln und zu betreuen, auch ein lohnendes Geschäftsfeld ist, was den Trend zum Haustarif weiter antreibt.

Der Verlust der Tarifbindung in weiten Bereichen des Handwerks verstärkt die seit Anfang der 1980er Jahre zu beobachtende zunehmende Abkoppelung der Einkommensentwicklung im Handwerk von der allgemeinen Lohnund Einkommensentwicklung. Für das Handwerk als Wirtschaftsbereich fördert die Erosion der Flächentarifverträge einen über Lohnsenkungsstrategien ausgetragenen Verdrängungswettbewerb, der langfristig die konstatierten Polarisierungstendenzen innerhalb des Handwerks vorantreiben wird: Ein Teil der Unternehmen wird in die Rolle des kostengünstigen Zulieferers bzw. Subunternehmers abgedrängt werden, mit entsprechend negativen Auswirkungen auf ihre Innova- tions- und Wettbewerbsfähigkeit sowie ihre Versorgung mit gut qualifizierten Fachkräften. Denn angesichts der zunehmend auseinanderklaffenden Lohnschere zu anderen Wirtschaftsbereichen und angesichts der schwächer werdenden Bindungswirkung einer diffuser werdenden Identität des Handwerks ist damit zu rechnen, dass in Zukunft die Abwanderungsneigung von Fachkräften aus dem Handwerk zunehmen wird.

Betriebsräte und Gewerkschaften sehen sich durch den Strukturwandel im Handwerk also stark veränderten Rahmenbedingungen gegenüber:

- Im Zuge von Polarisierungstendenzen im Handwerk und der Herausbildung eines sogenannten Großhandwerks entstehen Unternehmensgruppen mit mehreren Hundert bis mehreren Tausend Beschäftigten, die überregional bis bundesweit tätig sind und zentral gesteuert werden bzw. teilweise in Konzernstrukturen eingebunden sind, vor Ort aber durch kleinteilige, z.T. nicht stationäre Betriebs- und Arbeitsstrukturen gekennzeichnet sind und in ihrem Tätigkeitsfeld und Leistungsspektrum oft hergebrachte Gewerkegrenzen überschreiten.

- Der strukturellen Konzentration steht auf tarifpolitischer Seite eine Dezentralisierungstendenz gegenüber: eine Erosion der Flächentarifverträge, eine weit fortgeschrittene Verbetrieblichung der Tarifpolitik durch eine steigende Zahl von Haustarifverträgen und eine Fragmentierung der Tarifsituation innerhalb einzelner Unternehmensgruppen.

Diese Entwicklung findet vor dem Hintergrund einer nach wie vor schwach ausgebildeten Organisationsmacht der Gewerkschaften in diesem Wirtschaftssektor statt. So ist es den Gewerkschaften bisher nicht gelungen, im Handwerk stärker Fuß zu fassen, eher ist der Organisationsgrad in den letzten Jahren weiter gesunken. So hatte z. B. die IG Metall zwischen 2004 und 2012 im Handwerk einen Mitgliederrückgang von $9 \%$ zu verzeichnen.

Wie schlagen diese veränderten Rahmenbedingungen auf die Arbeit der betrieblichen und überbetrieblichen Interessenvertretungen im Handwerk durch? Welche neuen Aufgaben und Herausforderungen stellen sich mit dem Strukturwandel für Gewerkschaften und Betriebsräte im Handwerk?

3 Dies ist insbesondere ein Problem in den großen Unternehmensgruppen, die im Zuge der Entwicklung zum Full-ServiceAnbieter ihr Leistungsspektrum auf das technische Gebäudemanagement oder industrienahe Dienstleistungen ausgeweitet haben und die sich oft aus dem Gebäudereinigerhandwerk, großen Baukonzernen oder Instandhaltungsabteilungen großer Konzerne des Anlagenbaus entwickelt haben. Dort sind neben der IG Metall auch andere Einzelgewerkschaften wie die IG BCE, die IG Bau oder die Dienstleistungsgewerkschaft ver.di zuständig. Ein Ziel der IG Metall besteht daher darin, für gewerkeübergreifende Geschäftsfelder wie Facility Management oder industrienahe Dienstleistungen bundesweite, brancheneinheitlicheTarifverträge abzuschließen. 


\section{Anpassung an neue Unternehmens- strukturen}

Mit der Entwicklung zu Großbetrieben des Handwerks geht für die dortigen Interessenvertretungen häufig ein grundlegender Wandel des sozialen Umfelds einher, in dem sie agieren. Sie werden - durch Aufkauf oder Fusion - Teil einer oft unter dem Dach einer Managementholding zusammengefassten und zentral gelenkten Unternehmensgruppe. Insbesondere dort, wo solche Unternehmensgruppen aus inhabergeführten mittelständischen Handwerksbetrieben erwachsen sind und die Betriebsräte aus dieser Tradition der Interessenvertretungspraxis kommen, stellt die Anpassung an den damit verbundenen Wandel der „political culture“ der Interessenvertretungspraxis - formalisierter und verrechtlichter Arbeitsstil im Umgang mit dem Management, Konfrontation mit einem anderen Sozialtypus von Management mit Shareholder-Denken und geringer Betriebsbindung - ein nicht zu unterschätzendes Problem dar, das nicht selten erst durch einen Generationenwechsel in der Zusammensetzung der Betriebsratsgremien gelöst wird.

Angesichts des Wachstums ihrer Unternehmen sehen sich die Betriebsräte solcher Gruppen vor die Aufgabe gestellt, funktionierende betriebliche und überbetriebliche Mitbestimmungsstrukturen zu bilden. Wie kann in solchen Unternehmensgruppen des Handwerks mit einer klein- bis kleinstbetrieblichen Arbeits- und Betriebsorganisation und überregional verstreuten Standorten praktische Betriebsratsarbeit organisiert werden? Wie erreichen die Betriebsräte den Einbezug aller Standorte in die Interessenvertretungsorganisation und in die Betreuungsarbeit der Betriebsräte? Viele Standorte sind aufgrund ihrer Größe nicht betriebsratsfähig oder zu schwach, eigene Betriebsräte zu gründen. Dort, wo Betriebsräte existieren, verfügen sie häufig nicht über die Arbeitsvoraussetzungen, um wirkungsvolle Vertretungsarbeit leisten zu können. Eine auf einer örtlichen Definition des Betriebs basierende Betriebsratsorganisation ist kaum mehr möglich, wenn es, wie in den montierenden Dienstleistungshandwerken häufig der Fall, nur noch wenige feste Niederlassungen und keine stationäre Arbeitsorganisation mehr gibt, sondern in erster Linie mobile Montagegruppen, die je nach Kundenauftrag an wechselnden Standorten tätig sind.

Um zu großflächigeren Wahl- und Vertretungsbereichen mit arbeitsfähigen Betriebsratsgremien zu kommen, müssen die Interessenvertretungen einen der regionalen Struktur und den Entscheidungsstrukturen in ihrer Gruppe angemessenen, von örtlichen Standortstrukturen abgelösten Betriebsbegriff als Basis für die Organisation ihrer Betriebsratsstruktur entwickeln und durchsetzen. Das kann z. B. durch die Zusammenfassung von örtlichen Betriebsräten zu Regionalbetriebsräten geschehen, die es erlauben, zu größeren, effizienteren Gremien zu kommen, von denen einige evtl. eine Freistellung erreichen können. Wenn die Unternehmensgruppen in verschiedenen Geschäftsfeldern tätig sind, ist eine solche Zusammenfassung auf der Basis von Unternehmenssparten möglich, um den unterschiedlichen Problemen der Beschäftigten in verschiedenen Geschäftsfeldern gerecht zu werden.

Bei der Errichtung einer maßgeschneiderten, ihrer Unternehmensgruppe angepassten Betriebsratsorganisation sind die Interessenvertretungen jedoch nicht autonom. Für „verhandelte“ Betriebsratsstrukturen auf Basis einer tarifvertraglichen Regelung nach $₫ 3$ BetrVG sind sie auf den Konsens mit dem Arbeitgeber angewiesen. Gerade im Handwerk hat das Management jedoch häufig eher ein Interesse an kleinteiligen, schwachen Betriebsratsstrukturen. Das Fehlen von Selbstbestimmung der Interessenvertretung über ihre Betriebsratsstrukturen wirkt sich umso problematischer aus, als durch die ständigen Restrukturierungen in diesen sich formierenden Unternehmensgruppen (Aufspaltungen und Zusammenlegung von Betriebsteilen, Änderungen der Rechtsform der Gruppe oder einzelner Standorte) auch die bisherigen Grundlagen für die Bildung von betrieblichen und überbetrieblichen Betriebsratsstrukturen wiederholt infrage gestellt werden und dann neu verhandelt werden müssen - oft mit dem Ergebnis schwächerer Mitbestimmung.

Probleme resultieren allerdings nicht nur aus möglichen Widerständen des Managements, sondern auch aus den eigenen Reihen. So aus den Reihen der betroffenen Betriebsräte selbst, wenn es etwa bei der Zusammenfassung zu Regionalbetriebsräten zum Verlust von Mandaten kommt oder wenn kleinere Standorte um ihren Einfluss in den größeren Gremien fürchten oder auch vonseiten der Verwaltungsstellen, wenn die Betriebsratsgremien Verwaltungsstellengrenzen übergreifen und eine Verwaltungsstelle deshalb die Zuständigkeit und die Mitgliedsbeiträge für einzelne Standorte verliert.

Die konkreten Probleme des Aufbaus von passenden Betriebsratsstrukturen in solchen Unternehmensgruppen führen nicht selten dazu, dass in Handwerksgruppen problematische Mitbestimmungsstrukturen entstehen. So können funktionierende überbetriebliche Gremien ohne ausreichende Basis an örtlichen Betriebsräten bleiben, oder es können regional überdehnte Betriebsratsstrukturen entstehen. Das kann der Fall sein, wenn weit entfernte Standorte den Betriebsrat am Hauptstandort mitwählen ( $\$ 4$ BetrVG), anstatt eigene Betriebsräte oder Regionalbetriebsräte zu bilden. Solche Standorte können dann vom Betriebsrat aufgrund der Entfernung kaum betreut werden, und die Gewerkschaft hat dort wegen des Fehlens eines Standortbetriebsrats kaum Betriebszugang. 


\section{Bedeutungsgewinn überbetrieblicher Interessenvertretungsgremien}

Im Handwerk entstehen Unternehmensstrukturen, in denen komplexe, aus mehreren Ebenen bestehende Betriebsratsstrukturen (Gesamtbetriebsrat [GBR], Konzernbetriebsrat [KBR] oder auch neue Formen betriebs- und unternehmensübergreifender Betriebsratsstrukturen nach $\$ 3$ BetrVG) möglich sind und auch installiert werden. $24 \%$ der Betriebe im Handwerk hatten nach der WSI-Betriebsrätebefragung von 2004/2005 solche komplexen Betriebsratsstrukturen (Behrens 2005, S. 640), inzwischen dürften es deutlich mehr sein. GBR und KBR werden also auch im Handwerk an Bedeutung gewinnen und sind längst keine Angelegenheit von Großunternehmen mehr.

Angesichts der zunehmenden Auflösung tariflicher Makrostrukturen wächst zugleich die Bedeutung der überbetrieblichen Interessenvertretung als Standortinteressen koordinierende, Einheitlichkeit und Solidarität herstellende Instanz. Denn die Zersplitterung der tariflichen Situation in solchen Unternehmensgruppen führt $\mathrm{zu}$ oft massiven Differenzen bei Löhnen und anderen Arbeitsstandards zwischen den Beschäftigten der einzelnen Standorte. Für die Betriebsräte wächst damit das Risiko, dass Standorte gegeneinander ausgespielt werden: dass durch die Drohung, gruppeninterne Aufträge an „billigere“ Standorte zu vergeben, ein gruppeninterner Unterbietungswettbewerb in Gang gesetzt wird. Es kommt zu Entsolidarisierung und Spannungen zwischen den Belegschaften. Empirische Befunde aus dem Projekt zeigen denn auch, dass diese Rahmenbedingungen massiv auf die Arbeit der GBR in den Betrieben durchschlagen und es ihnen unter diesen Bedingungen häufig erschwert wird, eine betriebsübergreifende, unternehmensbezogene Interessenvertretungsperspektive auszubilden. Nicht selten werden die Potenziale eines GBR verschenkt, und die Interessenvertretungspraxis des GBR beschränkt sich dann auf bloßen Informationsaustausch. Es kann sogar zur vollständigen Blockade seiner Arbeit kommen.

Die gewachsene interessenpolitische Bedeutung des GBR als Koordinator unterschiedlicher Standortinteressen steht in krassem Gegensatz zu den restriktiven Bedingungen, unter denen GBR-Arbeit in diesem Umfeld häufig stattfinden muss. Die Reform des Betriebsverfassungsgesetzes ist in dieser Hinsicht unvollendet geblieben: Sie hat zwar die Bildung überbetrieblicher Interessenvertretungsgremien erleichtert, hat diese aber nicht mit angemessenen Arbeitsressourcen ausgestattet. Es gibt keine eigene Freistellung für die GBRArbeit. Oft verfügt angesichts der Standortstrukturen keiner der Standortbetriebsräte über eine Freistellung (allenfalls eine Teilfreistellung). Es gibt auch kein eigenes Recht auf Schulung für die GBR-Arbeit. Die Bewegungsmöglichkeiten des GBR-Vorsitzenden in Unternehmensgruppen mit überregional ausgedehnten Standortstrukturen und mobiler $\mathrm{Ar}$ beitsorganisation sind oft eingeschränkt, die Möglichkeiten, sich ausreichend häufig zu gemeinsamen GBR-Sitzungen zu treffen, sind reduziert. Damit fehlen die grundlegenden Voraussetzungen für eine wirksame, kontinuierliche und diesen Unternehmensnetzwerken adäquate überbetriebliche Interessenvertretungsarbeit. Die Sicherstellung von ausreichenden Arbeitsmöglichkeiten - z. B. im Rahmen einer die Bildung eines Betriebsrats nach $\$ 3$ BetrVG begleitenden Betriebsvereinbarung - gelingt selten und muss häufig erst in konfliktreichen Auseinandersetzungen durchgesetzt werden, denn das Management in diesen Unternehmensgruppen hat keinesfalls immer ein eigenes Interesse an der Installation eines kompetenten und arbeitsfähigen überbetrieblichen Verhandlungspartners und setzt oft schon der Bildung überbetrieblicher Gremien Widerstand entgegen.

\section{Betriebsräte als tarifpolitische Akteure}

Mit der Ausbreitung von Haustarifverträgen werden die Betriebsräte im Handwerk immer häufiger auch zu „tarifpolitischen Akteuren“ (Haipeter 2010), denn als Gewerkschaftsmitglieder sind sie automatisch Vertrauensleute ihrer Gewerkschaft, und der GBR ist in der Regel Mitglied der betrieblichen Tarif- und Verhandlungskommission.

Dies bedeutet zunächst neue Kompetenzanforderungen: Einarbeitung in komplexe tarifliche Regelungswerke, eine vertiefte Auseinandersetzung mit der wirtschaftlichen Lage und den Strategien des Unternehmens, steigende Anforderungen an die Kommunikationsfähigkeit und das diplomatische Verhandlungsgeschick. Dort, wo die Gesamtbetriebsräte über keine Freistellungen verfügen, die Standorte weit voneinander entfernt sind und die Arbeitsorganisation mobil ist, können mit einer Haustarifverhandlung, der notwendigen Koordination der Standortbetriebsräte und der anschließenden Umsetzung und Kontrolle des Tarifvertrags schnell die Grenzen der ohnehin knappen Ressourcen für Betriebsratsarbeit erreicht sein, was dann unter Umständen zulasten anderer Mitbestimmungsthemen geht.

Zum anderen geraten die Betriebsräte in Haustarifverhandlungen in neue Legitimationszwänge, da sie nun auch für Ergebnisse von Tarifverhandlungen gegenüber ihrer Belegschaft und den Gewerkschaftsmitgliedern geradestehen müssen. Und sie geraten in neue, spannungsreiche Rollenkonflikte: etwa zwischen der Rolle des auf den Betriebsfrieden verpflichteten Betriebsrats, der nach Abschluss der Verhandlungen wieder zu „normalen“ Arbeitsbeziehungen mit dem Arbeitgeber zurückfinden muss, und der Rolle als notfalls streikbereites Gewerkschaftsmitglied in einer Tarifauseinandersetzung; oder auch zwischen der Rolle als Standortbetriebsrat, der auf Wiederwahl durch seine Standortbelegschaft angewiesen ist und versuchen muss, möglichst viel für seine Belegschaft vor Ort herauszuholen, und der Rolle als GBR-Mitglied, das ein Interesse daran haben muss, das Lohngefälle abzubauen, um übergreifende Inte- 
ressenvertretungsarbeit leisten zu können, was meistens nicht ohne Zugeständnisse der bisher besser gestellten Standorte geht.

Neuen Anforderungen, Rollenkonflikten und Legitimationszwängen stehen aber auch neue Chancen gegenüber: Insbesondere dort, wo die Betriebsräte eher unerfahren sind, bergen Haustarifverhandlungen durch die Unterstützung der Gewerkschaft und die sich über längere Zeiträume hinziehende enge Zusammenarbeit mit deren Hauptamtlichen auch das Potenzial eines intensiven Lernprozesses für die Betriebsräte. Und im Erfolgsfall kann der Abschluss eines Haustarifvertrags die innerbetriebliche Position und das Prestige eines GBR außerordentlich stärken. Es sind oft die GBR großer, gut organisierter Handwerksgruppen, die bei der Gewerkschaft die Genehmigung zum Abschluss eines Haustarifvertrags beantragen. Für sie stellt ein Haustarif eine Möglichkeit dar, sich von der unterdurchschnittlichen Lohnentwicklung im Handwerk abzukoppeln und zugleich ihre GBR-Tätigkeit zu erleichtern.

Für die Gewerkschaft ist die Ausbreitung von Haustarifverträgen im Handwerk einerseits sehr problematisch. Denn angesichts der zentralen Bedeutung großer Unternehmensgruppen für die Tarifpolitik im Handwerk stellt das Ausscheren einer solchen Gruppe aus einer regionalen Tarifgemeinschaft immer auch eine Schwächung der tarifpolitischen Kampfkraft in der Fläche dar - nicht wenige Tarifauseinandersetzungen im Handwerk werden gegenwärtig nur noch durch die Streikkraft der Belegschaften einer solchen Unternehmensgruppe gewonnen. Ihr Ausscheren würde daher zu einem weiteren Absinken des Tariflohnniveaus im Handwerk führen und die Erosion des Flächentarifvertrags beschleunigen.

Andererseits bieten Haustarifverträge der Gewerkschaft auch Chancen. Erfolgreiche Haustarifverhandlungen führen in aller Regel zu Mitgliedergewinnen. Betriebsräte aus dem Handwerk sind außerdem bei der Aushandlung und Durchsetzung von Haustarifverträgen in der Regel auf die tarifpolitische Kompetenz und Unterstützung durch die Gewerkschaft angewiesen. Haustarifverhandlungen sind daher tendenziell geeignet, die Stellung der Gewerkschaft in den Unternehmensgruppen des Handwerks und ihre Verankerung unter den dortigen Betriebsräten zu stärken. Denn durch sie wird die bisherige Arbeitsteilung im Dualen System, die durch die strikte Trennung der Arenen „Tarifpolitik“ und „betriebliche Mitbestimmung" sowie eine hohe formale Unabhängigkeit der Betriebsräte von der Gewerkschaft gekennzeichnet war, von zwei Seiten her aufgebrochen: Die Betriebsräte werden zu tarifpolitischen Akteuren und die Gewerkschaft wird in Person ihres Hauptamtlichen - zumindest temporär - unmittelbar auf der Ebene des Betriebs präsent. Sie tritt dort dem Arbeitgeber als professioneller Verhandlungspartner auf Augenhöhe gegenüber eine betriebliche Präsenz der Gewerkschaft, die in Handwerksunternehmen keinesfalls selbstverständlich ist und Potenziale zur Veränderung des Mitbestimmungsklimas beinhaltet.

\section{Konsequenzen für die Gewerkschaften}

Der Blick auf die mit dem Strukturwandel ausgelösten Veränderungen zeigt, dass Betriebsräte und überbetriebliche Gremien im Handwerk zur Wahrnehmung ihrer immer komplexer werdenden Aufgaben auf die Unterstützung durch die Gewerkschaft angewiesen sind. Zweifelsohne verbindet sich mit der Herausbildung größerer Betriebseinheiten im Handwerk die Chance, mitbestimmungspolitisch stärker Fuß zu fassen. Zudem kommen infolge der Auslagerungen aus der Industrie gut organisierte Belegschaften aus Großunternehmen mit etablierter Mitbestimmungstradition in das Handwerk. Das kann eine - nicht zuletzt tarifpolitische - Stärkung des Handwerks bedeuten. Voraussetzung ist aber, dass die Gewerkschaft diese Betriebe und Belegschaften auch adäquat betreuen kann.

Genau diese Voraussetzung scheint derzeit nicht ausreichend gegeben. Vielmehr zeigt sich, dass eine traditionell ortsgebundene Betreuungs- und Zuständigkeitsstruktur der Gewerkschaftsorganisation mit dem Betreuungsbedarf der sich hier entwickelnden, verwaltungsstellen- und bezirksgrenzenüberschreitend tätigen Unternehmensgruppen und ihren netzwerkartigen Strukturen zunehmend weniger vereinbar ist:

- So sind für die Betreuung der Betriebsräte in den Standorten die örtlichen Verwaltungsstellen zuständig. Dort sind die Mitglieder registriert, dorthin fließen die Mitgliedsbeiträge. Die Unternehmensgruppen im Handwerk bestehen aber oft aus einer Vielzahl kleiner, bundesweit verstreuter Standorte. Obwohl diese Betriebsteile einer unter Umständen großen Unternehmensgruppe sind, ist ihr Mitgliederpotenzial vor Ort oft zu klein, um für die Verwaltungsstelle interessant zu sein.

- Für die Betreuung des GBR einer solchen Gruppe ist in der Regel der Sekretär der Verwaltungsstelle am Standort des GBR-Vorsitzenden zuständig. Die Betreuungsarbeit und die Teilnahme an GBR-Sitzungen sind für ihn häufig mit einem hohen Zeit- und Reiseaufwand verbunden - Kosten, die zulasten des Budgets seiner Verwaltungsstelle gehen. Die Mitgliedsbeiträge aus den anderen Standorten fließen aber an die örtlichen Verwaltungsstellen. Das führt zu Konflikten und zu Problemen, Sekretäre zu finden, die eine GBR-Betreuung übernehmen.

- Ungelöst ist das Problem der Koordinierung und Verknüpfung der Betreuung auf GBR-Ebene mit der Betreuung der einzelnen Standortbetriebsräte. Der Standortbetreuer muss wissen, was im GBR läuft, und umgekehrt.

- Schließlich erfordert eine qualifizierte Betreuung der Unternehmensgruppen den Aufbau von Branchenkompetenz auf zentraler Ebene: Wissen über die sich entwickelnden Branchen, ihre Dynamik und die hier agierenden wichtigsten Unternehmensgruppen. Und es muss dafür gesorgt werden, dass dieses Wissen an die betreuenden Sekretäre und Betriebsräte fließt. 
Die Entwicklung adäquater Betreuungsstrukturen würde in die bisherige Arbeitsteilung und Kompetenzverteilung zwischen den regionalen Ebenen der Gewerkschaft (Verwaltungsstelle, Bezirk, Zentrale) eingreifen. Sie müsste nicht zuletzt auch eine Neuverteilung der finanziellen Ressourcen zwischen den Gliederungen einschließen. Adäquate Betreuungsstrukturen würden erfordern, dass die Organisation der Betreuung nicht mehr automatisch örtlichen Zuordnungskriterien folgt, sondern der Struktur und Betriebsratsorganisation der jeweiligen Unternehmensgruppe. Auch die Erfassung und Zuordnung der Mitglieder und damit auch die Zuordnung der Mitgliedsbeiträge müsste dem gewählten Betreuungsmodell folgen.

Der Strukturwandel im Handwerk erfordert also einen parallelen organisatorischen Anpassungsprozess aufseiten der Gewerkschaft. Um die organisationspolitischen Chancen des Strukturwandels im Handwerk nutzen zu können, wäre letztlich das jetzige Muster der Allokation der Ressourcen zwischen Industrie und Handwerk generell zu hinterfragen. Dabei sollte man im Auge behalten: Wie die obige Analyse der Antriebskräfte des Strukturwandels gezeigt hat, sind die viel beschworenen Mitgliederpotenziale im Handwerk wenigstens zu einem gewissen Teil nur die Kehrseite abnehmender organisationspolitischer Potenziale in den Kernbereichen von industriellen Großunternehmen, die Dienstleistungen ins Handwerk auslagern.

\section{LITERATUR}

Behrens, M. (2005): Die Arbeit des Betriebsrats in komplexen Gremienstrukturen, in:WSI-Mitteilungen 58 (11), S. 638, http://www.boeckler.de/wsimit_2005_ 11_behrens.pdf

Haipeter, Th. (2010): Betriebsräte als neue Tarifakteure: Zum Wandel der Mitbestimmung beiTarifabweichungen, Forschung aus der Hans-Böckler-Stiftung, Bd. 114, Berlin

handwerk-magazin (2011): Die Top 1000 des Deutschen Handwerks, 3. August, http://www.handwerk-magazin.de/die-top-1000-des-deutschen-handwerks/ 150/13/39706/

IG Metall-Vorstand (2011): Diskussionspapier zu tarif- und organisationspolitischen Herausforderungen im Handwerk, Frankfurt a. M.

Müller, K. (2012): Analyse der Handwerkszählung 2008, Göttinger Handwerkswirtschaftliche Studien, Bd. 85, Duderstadt

\section{AUTORIN}

STEFANIE WEIMER, Dr., Wissenschaftliche Mitarbeiterin am Institut für Sozialwissenschaftliche Forschung (ISF), München. Arbeitsschwerpunkte: Arbeit und Strukturwandel in KMU und Handwerk, Arbeitswelt und demografischer Wandel, Arbeitsgestaltung und Personalentwicklung.

@ stefanie.weimer@isf-muenchen.de 